PACS: 63.20.-e, 63.20.Ry, 05.30.Jp

\title{
ON DESCRIPTION OF THERMODYNAMIC PROPERTIES OF CRYSTALS IN THE APPROXIMATION OF AN ISOTROPIC MEDIUM. THE TWO-PARAMETRIC DEBYE MODEL
}

\author{
Yu.M. Poluektov 1,2 \\ ${ }^{1}$ National Science Center "Kharkov Institute of Physics and Technology" \\ 1, Akademicheskaya Str., 61108 Kharkov, Ukraine \\ ${ }^{2}$ Kharkov V.N. Karazin National University \\ Sq. Svobody 4, Kharkov, 61022, Ukraine \\ E-mail:yuripoluektov@kipt.kharkov.ua,https://orcid.org/0000-0002-3207-3226 \\ Received June 18, 2018; accepted July 26, 2018
}

When analyzing thermodynamic and kinetic properties of crystals whose anisotropy is not large and the considered effects do not relate to the existence of singled-out directions in crystals, one may use a more simple model of an isotropic medium with a good accuracy, after having chosen its parameters in an optimal way. Based on the quantum mechanical description it is shown that the method of approximation of the moduli of elasticity of a crystal by the model of an isotropic medium, proposed earlier in [2], follows from the requirement of the minimal difference between the free energies of a crystal and an approximating isotropic medium. The two-parametric Debye model is formulated, which, in contrast to the standard model where the average speed of phonons is introduced, takes into account the existence in an isotropic medium of both longitudinal and transverse phonons. The proposed model contains, except the Debye energy, an additional dimensionless parameter and, consequently, the law of corresponding states for the heat capacity being characteristic of the standard model does not hold. With taking account of the two phonon branches the structure of the density of phonon states proves to be more complex as compared to the standard model and has a singularity that resembles Van Hove singularities in real crystals. As an example, an application of the two-parametric Debye theory to such crystals of the cubic system as tungsten, copper, lead is considered. It is shown that the calculation of the low-temperature heat capacity of these crystals by means of the approximated moduli of elasticity within the framework of the two-parametric model leads to a considerably better agreement with experiment than in the case of the standard Debye model.

KEY WORDS: moduli of elasticity, phonon, free energy, heat capacity, Debye energy

\author{
ПРО ОПИС ТЕРМОДИНАМІЧНИХ ВЛАСТИВОСТЕЙ КРИСТАЛІВ \\ У НАБЛИЖЕННІ ІЗОТРОПНОГО СЕРЕДОВИЩА. ДВОПАРАМЕТРИЧНА МОДЕЛЬ ДЕБАЯ \\ Ю.М. Полуектов ${ }^{1,2}$ \\ ${ }^{1}$ Національний науковий центр “Харківський фізико-технічний інститут” \\ 61108, вул. Академічна, 1, Харків, Україна \\ ${ }^{2}$ Харківський начіональний університет ім. В.Н. Каразіна \\ пл. Свободи, 4, м Харків, 61022, Україна
}

При аналізі термодинамічних і кінетичних властивостей кристалів, анізотропія яких не $є$ великою, а ефекти що розглядаються не пов'язані з існуванням у кристалах виділених напрямків, можна з хорошою точністю користуватися більш простою моделлю ізотропного середовища, підібравши ії параметри оптимальним чином. На основі квантово-механічного розгляду показано, що метод апроксимації модулів пружності кристала моделлю ізотропного середовища, запропонований раніше в [2], випливає 3 вимоги мінімальної різниці між вільними енергіями кристала і апроксимуючого ізотропного середовища. Сформульовано двохпараметричну модель Дебая, яка, на відміну від стандартної моделі, де вводиться середня швидкість фононів, враховує існування в ізотропному середовищі як поздовжніх, так і поперечних фононів. У запропонованій моделі крім енергії Дебая існує додатковий безрозмірний параметр i, внаслідок цього, закон відповідних станів для теплоємності, характерний для стандартної моделі, не виконується. При урахуванні двох фононних гілок структура густини фононних станів виявляється складнішою, ніж в стандартній моделі і має особливість, що нагадує особливості Ван Хова в реальних кристалах. Як приклад розглянуто застосування двохпараметричної теорії Дебая до таких кристалів кубічної сингонії, як вольфрам, мідь і свинець. Показано, що розрахунок низькотемпературної теплоємності цих кристалів за допомогою апроксимованих модулів пружності в рамках двохпараметричної моделі призводить до значно кращої згоди з експериментом, ніж у разі стандартної моделі Дебая.

КЛЮЧОВІ СЛОВА: модулі пружності, фонон, вільна енергія, теплоємність, енергія Дебая

\section{ОБ ОПИСАНИИ ТЕРМОДИНАМИЧЕСКИХ СВОЙСТВ КРИСТАЛЛОВ В ПРИБЛИЖЕНИИ ИЗОТРОПНОЙ СРЕДЫ. ДВУХПАРАМЕТРИЧЕСКАЯ МОДЕЛЬ ДЕБАЯ Ю.М. Полуэктов 1,2 \\ ${ }^{1}$ Национальный научный центр "Харьковский физико-технический институт" 61108, Академическая, 1, Харьков, Украина \\ ${ }^{2}$ Харьковский национальный университет им. В.Н. Каразина пл. Свободыл, 4, г. Харьков, 61022, Украина}

При анализе термодинамических и кинетических свойств кристаллов, анизотропия которых не велика, а рассматриваемые эффекты не связаны с существованием в кристаллах выделенных направлений, можно с хорошей точностью пользоваться более простой моделью изотропной среды, подобрав её параметры оптимальным образом. На основе квантово-механического рассмотрения показано, что метод аппроксимации модулей упругости кристалла моделью изотропной среды, предложенный ранее в [2], следует из требования минимальной разности между свободными энергиями кристалла и аппроксимирующей изотропной среды. 
Сформулирована двухпараметрическая модель Дебая, которая, в отличие от стандартной модели, где вводится средняя скорость фононов, учитывает существование в изотропной среде как продольных, так и поперечных фононов. В предложенной модели помимо энергии Дебая имеется дополнительный безразмерный параметр и, вследствие этого, закон соответственных состояний для теплоёмкости, характерный для стандартной модели, не выполняется. При учёте двух фононных ветвей структура плотности фононных состояний оказывается сложнее, чем в стандартной модели и имеет особенность, напоминающую особенности Ван Хова в реальных кристаллах. В качестве примера рассмотрено применение двухпараметрической теории Дебая к таким кристаллам кубической сингонии, как вольфрам, медь и свинец. Показано, что расчет низкотемпературной теплоемкости этих кристаллов с помощью аппроксимированных модулей упругости в рамках двухпараметрической модели приводит к значительно лучшему согласию с экспериментом, чем в случае стандартной модели Дебая.

КЛЮЧЕВЫЕ СЛОВА: модули упругости, фонон, свободная энергия, теплоемкость, энергия Дебая

Упругие свойства изотропной среды характеризуются двумя модулями упругости, например, коэффициентами Ламэ $\lambda$ и $\mu$ [1]. Кристалл является анизотропной средой, в силу чего его упругие свойства отличаются от свойств изотропного тела и характеризуются большим числом параметров. Если же величина анизотропии достаточно мала, то, очевидно, что свойства кристалла будут незначительно отличаться от свойств изотропной среды и анализ свойств кристалла можно заменить более простым анализом свойств модельной изотропной среды, подобрав её параметры оптимальным образом. В работе [2] модули упругости аппроксимирующего изотропного тела предложено находить из условия минимума величины

$$
G \equiv\left(\lambda_{i k l m}-\lambda_{i k l m}^{(0)}\right)^{2}
$$

где $\lambda_{i k l m}$ - тензор модулей упругости кристалла, а

$$
\lambda_{i k l m}^{(0)}=\lambda \delta_{i k} \delta_{l m}+\mu\left(\delta_{i l} \delta_{k m}+\delta_{i m} \delta_{k l}\right)
$$

- тензор модулей упругости изотропной среды. Условия экстремума $\partial G / \partial \lambda=\partial G / \partial \mu=0$ дают связь аппроксимирующих коэффициентов Ламэ с инвариантами тензора модулей упругости кристалла:

$$
\lambda=\frac{1}{15}\left(2 \lambda_{i k k}-\lambda_{i k i k}\right), \quad \mu=\frac{1}{30}\left(3 \lambda_{i k i k}-\lambda_{i i k k}\right) .
$$

Данная аппроксимация вполне разумна для качественного, а во многих случаях и количественного, анализа интегральных свойств кристалла и тех эффектов, которые не связаны с существованием в кристаллах выделенных направлений и могут быть описаны в приближении изотропной среды. В [2] такой подход был развит для теории упругих волн в кристаллах. Аналогичный подход может быть использован и при учете нелинейных эффектов в кристаллах, например, для упрощения расчётов матричных элементов взаимодействия фононов в кристаллах любой сингонии [3,4]. В данной работе ограничимся рамками линейной теории упругости.

Условие (1) удобно тем, что приводит к системе линейных алгебраических уравнений, однако в принципе возможны и другие критерии близости тензоров модулей упругости реальной и модельной сред, содержащие, например, модуль разности в более высокой степени. Естественно потребовать, чтобы аппроксимирующие модули упругости выбирались так, чтобы свободные энергии реальной и модельной сред были максимально близки.

Цель данной работы показать на основе квантового рассмотрения, что модули упругости изотропной модельной среды, полученные из условия экстремума величины $G(1)$, действительно приводят к свободной энергии, максимально близкой к свободной энергии кристалла.

Для описания термодинамических свойств твердых тел в приближении изотропной среды широко используется известная модель Дебая [5]. В стандартной модели Дебая, которая формулируется для изотропной среды, делается дальнейшее упрощение, состоящее в том, что изотропная среда вместо двух параметров, характеризуется единственным параметром - средней скоростью фононов и соответствующей ей энергией. Однако такое упрощение не является необходимым и представляется естественным формулировать теорию для изотропной среды с двумя модулями упругости и, соответственно, с двумя типами фононов - продольными и поперечными. Очевидно, что такая теория не может быть менее точной, чем модель Дебая в стандартной формулировке [5], и, возможно, позволит описать некоторые более тонкие эффекты. Кроме того, пользуясь, аппроксимацией модулей упругости (3), появляется возможность применить модель к расчету кристаллов различных сингоний. Таким образом, еще одной целью данной работы является формулирование модели Дебая, в которой учтено существование в изотропной среде продольных и поперечных фононов. В качестве примера рассмотрено применение предложенной двухпараметрической теории Дебая к кристаллам кубической сингонии. Найдены аппроксимирующие модули упругости для вольфрама, меди и свинца. Расчет их низкотемпературных теплоемкостей с использованием рассчитанных модулей показывает, что двухпараметрическая модель описывает термодинамические свойства с гораздо лучшим приближением, чем стандартная модель Дебая.

\section{ОПТИМАЛЬНАЯ АППРОКСИМАЦИЯ УПРУГИХ СВОЙСТВ КРИСТАЛЛА МОДЕЛЬЮ ИЗОТРОПНОЙ СРЕДЫ}

Плотность гамильтониана кристалла, как упругой среды, даётся выражением

$$
\mathrm{H}(\mathbf{r})=\frac{\pi_{a}(\mathbf{r})^{2}}{2 \rho}+\frac{1}{2} \lambda_{a i b j} u_{a i}(\mathbf{r}) u_{b j}(\mathbf{r}),
$$


где тензор деформации в линейном приближении имеет вид

$$
u_{i j}=\frac{1}{2}\left(\nabla_{j} u_{i}+\nabla_{i} u_{j}\right),
$$

$u_{i}(\mathbf{r})$ - вектор смещения, $\pi_{a}(\mathbf{r})=\rho \dot{u}_{a}(\mathbf{r})$ - канонический импульс, $\rho$ - плотность, $\lambda_{a i b j}$ - тензор модулей упругости кристалла. В (4) и в дальнейшем используется соглашение о суммировании по повторяющимся индексам.

Попытаемся аппроксимировать гамильтониан (4), содержащий модули упругости реального кристалла модельной изотропной средой, выбрав её гамильтониан в виде

$$
\mathrm{H}_{S}(\mathbf{r})=\frac{\pi_{a}(\mathbf{r})^{2}}{2 \rho}+\frac{1}{2} \tilde{\lambda}_{a i b j} u_{a i}(\mathbf{r}) u_{b j}(\mathbf{r})+\varepsilon_{0},
$$

где

$$
\tilde{\lambda}_{a i b j}=\tilde{\lambda} \delta_{a i} \delta_{b j}+\tilde{\mu}\left(\delta_{a b} \delta_{i j}+\delta_{a j} \delta_{b i}\right),
$$

а коэффициенты Ламэ $\tilde{\lambda}, \tilde{\mu}$ будем рассматривать как параметры, определяемые из условия наилучшей аппроксимации гамильтониана кристалла (4) гамильтонианом изотропной среды (6), которое будет введено ниже. Здесь и дальше модули упругости аппроксимирующей изотропной среды будем обозначать со значком «тильда» вверху. Гамильтониан (6) содержит плотность энергии $\varepsilon_{0}$, которая обусловлена тем, что замена точного тензора упругости $\lambda_{\text {aibj }}$ приближенным изотропным $\tilde{\lambda}_{a i b j}$ может приводить, вообще говоря, к изменению и основного недеформированного состояния кристалла. Полный исходный гамильтониан $H=\int \mathrm{H}(\mathbf{r}) d \mathbf{r}$ может быть представлен в виде $H=H_{S}+H_{C}$, где выделен полный аппроксимирующий гамильтониан:

$$
H_{S}=\int\left[\frac{\pi_{a}(\mathbf{r})^{2}}{2 \rho}+\frac{1}{2} \tilde{\lambda}_{a i b j} u_{a i}(\mathbf{r}) u_{b j}(\mathbf{r})\right] d \mathbf{r}+V \varepsilon_{0},
$$

а корреляционный гамильтониан

$$
H_{C}=\frac{1}{2} \int\left[\left(\lambda_{\text {aibj }}-\tilde{\lambda}_{\text {aibj }}\right) \nabla_{i} u_{a} \nabla_{j} u_{b}\right] d \mathbf{r}-V \varepsilon_{0}
$$

характеризует различие между точным и аппроксимирующим гамильтонианом.

При квантовом описании, которым будем пользоваться, вектор деформации $u_{a}(\mathbf{r})$ и канонический импульс $\pi_{a}(\mathbf{r})$ следует рассматривать как операторы, для которых справедливы известные соотношения коммутации

$$
\begin{gathered}
\pi_{a}(\mathbf{r}) u_{b}\left(\mathbf{r}^{\prime}\right)-u_{b}\left(\mathbf{r}^{\prime}\right) \pi_{a}(\mathbf{r})=-i \hbar \delta_{a b} \delta\left(\mathbf{r}-\mathbf{r}^{\prime}\right), \\
u_{a}(\mathbf{r}) u_{b}\left(\mathbf{r}^{\prime}\right)-u_{b}\left(\mathbf{r}^{\prime}\right) u_{a}(\mathbf{r})=0, \quad \pi_{a}(\mathbf{r}) \pi_{b}\left(\mathbf{r}^{\prime}\right)-\pi_{b}\left(\mathbf{r}^{\prime}\right) \pi_{a}(\mathbf{r})=0 .
\end{gathered}
$$

Воспользуемся разложением полевых операторов

$$
u_{a}(\mathbf{r})=\frac{1}{\sqrt{V}} \sum_{k, \alpha} \sqrt{\frac{\hbar}{2 \rho \omega(\mathbf{k}, \alpha)}} e_{a}(\mathbf{k}, \alpha)\left(b_{k \alpha}+b_{-k \alpha}^{+}\right) e^{i \mathbf{k r}}, \pi_{a}(\mathbf{r})=-\frac{i}{\sqrt{V}} \sum_{k, \alpha} \sqrt{\frac{\rho \hbar \omega(\mathbf{k}, \alpha)}{2}} e_{a}(\mathbf{k}, \alpha)\left(b_{k \alpha}-b_{-k \alpha}^{+}\right) e^{i \mathbf{k r}},
$$

где $\mathbf{e}(\mathbf{k}, \alpha)$ - комплексные векторы поляризации $(\alpha=1,2,3)$, такие что $\mathbf{e}(-\mathbf{k}, \alpha)=\mathbf{e}^{*}(\mathbf{k}, \alpha)$, для которых выполнены условия ортогональности и полноты

$$
\mathbf{e}(\mathbf{k}, \alpha) \mathbf{e}^{*}\left(\mathbf{k}, \alpha^{\prime}\right)=\delta_{\alpha \alpha^{\prime}}, \quad \sum_{\alpha} e_{i}^{*}(\mathbf{k}, \alpha) e_{j}(\mathbf{k}, \alpha)=\delta_{i j}
$$

Операторы рождения $b_{k \alpha}^{+}$и уничтожения $b_{k \alpha}$ фононов подчиняются обычным условиям коммутации: $\left[b_{k \alpha}, b_{k^{\prime} \alpha^{\prime}}^{+}\right]=\delta_{k k^{\prime}} \delta_{\alpha \alpha^{\prime}},\left[b_{k \alpha}, b_{k^{\prime} \alpha^{\prime}}\right]=\left[b_{k \alpha}^{+}, b_{k^{\prime} \alpha^{\prime}}^{+}\right]=0$. При описании изотропной среды один из векторов поляризации удобно выбрать вдоль волнового вектора: $\mathbf{e}(\mathbf{k}, 3)=i(\mathbf{k} / k)=i \mathbf{n}$. Два других вектора поляризации с $\alpha=1,2$ лежат в плоскости перпендикулярной волновому вектору. С учетом этого аппроксимирующий гамильтониан (8) может быть записан диагональной форме

$$
H_{S}=\sum_{k}\left(2 \hbar \omega_{k t} b_{k t}^{+} b_{k t}+\hbar \omega_{k l} b_{k l}^{+} b_{k l}\right)+\sum_{k}\left(\hbar \omega_{k t}+\frac{1}{2} \hbar \omega_{k l}\right)+V \varepsilon_{0} .
$$

Здесь фононы с поперечной поляризацией с $\alpha=1,2$ обозначены одним индексом $t$, а фононы с продольной поляризацией с $\alpha=3$ - индексом $l$. Также учтено, что поперечные фононы имеют две степени свободы. В 
результате приведения гамильтониана к диагональной форме (13), находим законы дисперсии фононов $\omega_{k t}=c_{t} k, \omega_{k l}=c_{l} k$, где скорости поперечных и продольных фононов даются известными выражениями [1]:

$$
c_{t}=\sqrt{\frac{\tilde{\mu}}{\rho}}, \quad c_{l}=\sqrt{\frac{\tilde{\lambda}+2 \tilde{\mu}}{\rho}} .
$$

В дальнейшем вычисления средних величин будем проводить с помощью статистического оператора

$$
\hat{\rho}=\exp \beta\left(F-H_{S}\right),
$$

где $\beta=1 / T$ - обратная температура. Условие нормировки $\mathrm{Sp} \hat{\rho}=1$ приводит к формуле для свободной энергии в модели самосогласованного поля

$$
F=V \varepsilon_{0}+\sum_{k}\left(\hbar \omega_{k t}+\frac{1}{2} \hbar \omega_{k l}\right)+2 T \sum_{k} \ln \left(1-e^{-\beta \hbar \omega_{k l}}\right)+T \sum_{k} \ln \left(1-e^{-\beta \hbar \omega_{k l}}\right) .
$$

Энергия недеформированного состояния $\varepsilon_{0}$ в такой модели находится из условия равенства средних для точного и аппроксимирующего гамильтонианов $\langle H\rangle=\left\langle H_{S}\right\rangle$, или, что эквивалентно, из условия $\left\langle H_{C}\right\rangle=0$. Это дает

$$
\varepsilon_{0}=\frac{1}{2 V} \int\left[\left(\lambda_{\text {aibj }}-\tilde{\lambda}_{\text {aibj }}\right)\left\langle\nabla_{i} u_{a} \nabla_{j} u_{b}\right\rangle\right] d \mathbf{r} .
$$

Вычисляя среднее в (17) с помощью статистического оператора (15), приходим к следующей формуле

$$
\varepsilon_{0}=\frac{\hbar}{4 \rho V}\left(\lambda_{\text {aibj }}-\tilde{\lambda}_{\text {aibj }}\right) \sum_{k} k_{i} k_{j}\left[A_{k t} \delta_{a b}+\left(A_{k l}-A_{k t}\right) n_{a} n_{b}\right],
$$

где величины

$$
A_{k t}=\frac{1+2 f_{k t}}{\omega_{k t}}, \quad A_{k l}=\frac{1+2 f_{k l}}{\omega_{k l}}
$$

выражаются через функции распределения продольных $f_{k l}=\left[\exp \beta \hbar \omega_{k l}-1\right]^{-1}$ и поперечных $f_{k t}=\left[\exp \beta \hbar \omega_{k t}-1\right]^{-1}$ фононов. Входящие в (18) суммы по волновым векторам, переходя от суммирования к интегрированию и интегрируя по углам, можно представить в виде

$$
\sum_{k} k_{i} k_{j} A_{k t}=\frac{V}{6 \pi^{2}} \delta_{i j} I_{t}, \quad \sum_{k} k_{i} k_{j} n_{a} n_{b}\left(A_{k l}-A_{k t}\right)=\frac{V}{30 \pi^{2}}\left(\delta_{i j} \delta_{a b}+\delta_{i a} \delta_{j b}+\delta_{i b} \delta_{j a}\right)\left(I_{t}-I_{l}\right),
$$

где

$$
I_{t}=\int_{0}^{k_{D}} d k k^{4} \frac{\left(1+2 f_{k t}\right)}{\omega_{k t}}=\frac{k_{D}^{4}}{4 c_{t}} \Phi\left(\frac{\Theta_{t}}{T}\right), \quad I_{l}=\int_{0}^{k_{D}} d k k^{4} \frac{\left(1+2 f_{k l}\right)}{\omega_{k l}}=\frac{k_{D}^{4}}{4 c_{l}} \Phi\left(\frac{\Theta_{l}}{T}\right) .
$$

Здесь $\Theta_{t}=\hbar c_{t} k_{D}, \Theta_{l}=\hbar c_{l} k_{D}$ - энергии Дебая, определённые через скорости поперечных и продольных фононов, $k_{D}=\left(6 \pi^{2} N / V\right)^{1 / 3}$ - волновое число Дебая, $N$ - число частиц. В (21) определена функция

$$
\Phi(x)=1+(8 / 3 x) D(x), \quad D(x)=\frac{3}{x^{3}} \int_{0}^{x} \frac{z^{3} d z}{e^{z}-1} .
$$

В результате находим

$$
\varepsilon_{0}=\frac{3 N T}{16 V}\left[\left(\frac{\lambda_{l}}{x_{l}}-x_{l}\right) \Phi\left(x_{l}\right)+2\left(\frac{\lambda_{t}}{x_{t}}-x_{t}\right) \Phi\left(x_{t}\right)\right],
$$

где для краткости введены обозначения:

$$
\begin{gathered}
x_{l} \equiv \frac{\Theta_{l}}{T}=\frac{\hbar c_{l} k_{D}}{T}, \quad x_{t} \equiv \frac{\Theta_{t}}{T}=\frac{\hbar c_{t} k_{D}}{T}, \\
\lambda_{l} \equiv \frac{\hbar^{2} k_{D}^{2}}{15 \rho T^{2}}\left(2 \lambda_{B}+\lambda_{A}\right), \quad \lambda_{t} \equiv \frac{\hbar^{2} k_{D}^{2}}{30 \rho T^{2}}\left(3 \lambda_{B}-\lambda_{A}\right) .
\end{gathered}
$$

Здесь $\lambda_{A} \equiv \lambda_{\text {iaa }}, \lambda_{B} \equiv \lambda_{\text {aiai }}$ - инварианты тензора модулей упругости кристалла. В результате, с учетом (16), получим свободную энергию, как функцию температуры, объёма, числа частиц и двух параметров $x_{l}, x_{t}$ :

$$
\begin{aligned}
F & =\frac{3 N T}{16}\left[\Phi\left(x_{l}\right)\left(\frac{\lambda_{l}}{x_{l}}-x_{l}\right)+2 \Phi\left(x_{t}\right)\left(\frac{\lambda_{t}}{x_{t}}-x_{t}\right)\right]+\frac{3 N T}{8}\left(x_{l}+2 x_{t}\right)+ \\
& +\frac{N T}{3}\left[3 \ln \left(1-e^{-x_{l}}\right)-D\left(x_{l}\right)\right]+\frac{2 N T}{3}\left[3 \ln \left(1-e^{-x_{l}}\right)-D\left(x_{t}\right)\right] .
\end{aligned}
$$


При произвольных значениях параметров $x_{l}, x_{t}$ свободная энергия (25) описывает систему в состоянии с неполным термодинамическим равновесием. Равновесные значения этих параметров, а, следовательно, и оптимальные величины коэффициентов упругости модельной изотропной среды $\tilde{\lambda}$ и $\tilde{\mu}$, следует находить из условий экстремума свободной энергии (25):

$$
\frac{\partial F}{\partial x_{l}}=0, \quad \frac{\partial F}{\partial x_{t}}=0 .
$$

В результате получим уравнения:

$$
\left(\frac{\lambda_{\alpha}}{x_{\alpha}^{2}}-1\right)\left[x_{\alpha} \Phi^{\prime}\left(x_{\alpha}\right)-\Phi\left(x_{\alpha}\right)\right]=0,
$$

где $\alpha=l, t$. Функция во вторых скобках в области своего определения не обращается в нуль, так что должно быть $x_{\alpha}^{2}=\lambda_{\alpha}$ или более подробно:

$$
c_{l}^{2}=\frac{1}{15 \rho}\left(2 \lambda_{B}+\lambda_{A}\right), \quad c_{t}^{2}=\frac{1}{30 \rho}\left(3 \lambda_{B}-\lambda_{A}\right) .
$$

Отсюда, согласно (14), формулы для коэффициентов Ламэ изотропной среды, моделирующей кристалл, имеют вид

$$
\tilde{\lambda}=\frac{1}{15}\left(2 \lambda_{A}-\lambda_{B}\right), \quad \tilde{\mu}=\frac{1}{30}\left(3 \lambda_{B}-\lambda_{A}\right) .
$$

Эти формулы совпадают с формулами (3), полученными на основе иного критерия, введенного в [2]. Как было здесь также показано, подобная аппроксимация оказывается справедливой при произвольной температуре. Приведем аппроксимирующие формулы для модуля всестороннего сжатия $\tilde{K}$, модуля Юнга $\tilde{E}$ и коэффициента Пуассона $\tilde{\sigma}$ :

$$
\tilde{K}=\frac{1}{9} \lambda_{A}, \quad \tilde{E}=\frac{\lambda_{A}\left(3 \lambda_{B}-\lambda_{A}\right)}{3\left(3 \lambda_{A}+\lambda_{B}\right)}, \quad \tilde{\sigma}=\frac{2 \lambda_{A}-\lambda_{B}}{3 \lambda_{A}+\lambda_{B}} .
$$

Обратим внимание, что модуль сжатия аппроксимирующей сплошной среды определяется только одним инвариантом кристалла $\lambda_{A} \equiv \lambda_{\text {iiaa }}$.

\section{ДВУХПАРАМЕТРИЧЕСКАЯ МОДЕЛЬ ДЕБАЯ}

Модель Дебая [5] описывает термодинамические свойства изотропной упругой среды. Упругие свойства такой среды характеризуются, двумя модулями упругости, в качестве которых можно выбрать, например, коэффициенты Ламэ $\lambda$ и $\mu$ или какие-либо два других модуля (30). В соответствии с этим имеется две звуковые моды, отвечающие продольным и поперечным колебаниям и две скорости $c_{l}$ и $c_{t}$ распространения таких волн (14). В стандартной теории Дебая [5] используется еще одно упрощение: вместо двух скоростей, вводится средняя скорость звуковых колебаний $c_{D}$, определяемая соотношением:

$$
\frac{1}{c_{D}^{3}}=\frac{2}{c_{t}^{3}}+\frac{1}{c_{l}^{3}}=\rho^{3 / 2}\left[\frac{2}{\mu^{3 / 2}}+\frac{1}{(\lambda+2 \mu)^{3 / 2}}\right] .
$$

Следствием этого приближения является то, что изотропная упругая среда характеризуется единственным параметром $\Theta_{D}=\hbar c_{D} k_{D}$ - энергией Дебая, а теплоемкость оказывается функцией только отношения $T / \Theta_{D}$. Определение (31) означает, что фактически усредняются обратные кубы скоростей. Однако, определить среднюю скорость можно и иначе, например, соотношением

$$
c_{0}^{2}=\frac{1}{3}\left(2 c_{t}^{2}+c_{l}^{2}\right)=\frac{(\lambda+4 \mu)}{3 \rho},
$$

которое возникает в подходе, основанном на описании взаимодействующих фононов в модели самосогласованного поля [6,7]. Определение (32) представляется более естественным, поскольку при $\mu \rightarrow 0$ и $c_{t} \rightarrow 0$ из (31) следует, что и $c_{D} \rightarrow 0$, тогда как определение (32) дает в этом случае конечное значение средней скорости $c_{0}$. Отношение средних скоростей, определенных формулами (31) и (32) зависит от отношения коэффициентов Ламэ

$$
\frac{c_{0}^{2}}{c_{D}^{2}}=\frac{1}{3^{5 / 3}}(4+\lambda / \mu)\left[2+\frac{1}{(2+\lambda / \mu)^{3 / 2}}\right]^{2 / 3},
$$

где $\lambda / \mu>-2 / 3$ [1]. Зависимость отношения скоростей $c_{D} / c_{0}$ от отношения $\lambda / \mu$ показана на рис. 1 . С увеличением отношения $\lambda / \mu$ различие в средних скоростях, определённых формулами (31) и (32) возрастает. Может возникнуть вопрос, какое усреднение считать правильным. Если считать среднюю скорость и энергию Дебая феноменологическими параметрами, то этот вопрос, по-видимому, не является принципиальным. В тоже время, очевидно, что переход от использования двух параметров, которыми характеризуется упругая среда, к 
одному параметру не является необходимым и более естественно строить теорию с учетом обеих скоростей фононов. При этом усложнение теории оказывается незначительным, а вопрос о способе введения усредненной скорости вообще не возникает. Можно предположить, что теория с двумя энергиями Дебая будет иногда описывать более тонкие эффекты. Кроме того, появляется возможность применения такой модели для расчетов кристаллов различной симметрии с помощью модулей упругости, если пользоваться методом аппроксимирующей изотропной среды, который был рассмотрен выше.

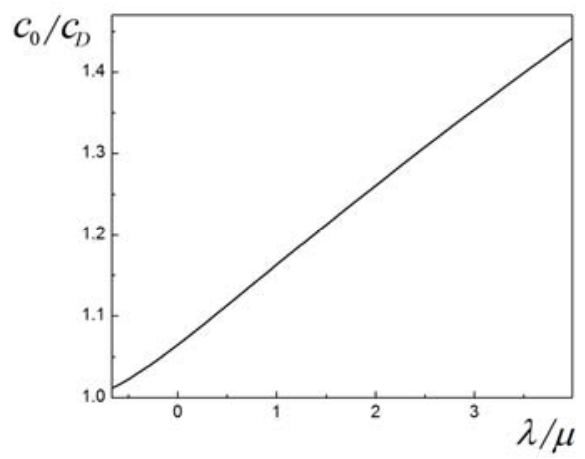

Рис. 1. Зависимость отношения средних скоростей фононов $c_{0} / c_{D}$, определенных формулами (31) и (32), от отношения коэффициентов Ламэ $\lambda / \mu$

В этом разделе сформулируем модель Дебая, не пользуясь усреднением скорости фононов. Такую модель естественно назвать двухпараметрической. Обычную модель Дебая будем также называть однопараметрической. При оптимальном выборе скоростей фононов (28) свободная энергия принимает вид

$$
F=\frac{3 N}{8}\left(\Theta_{l}+2 \Theta_{t}\right)+\frac{N T}{3}\left[3 \ln \left(1-e^{-\beta \Theta_{l}}\right)-D\left(\beta \Theta_{l}\right)\right]+\frac{2 N T}{3}\left[3 \ln \left(1-e^{-\beta \Theta_{t}}\right)-D\left(\beta \Theta_{t}\right)\right] .
$$

Энтропия $S=-\partial F / \partial T$ и энергия $E=F+T S$ даются формулами:

$$
\begin{gathered}
S=-\frac{N}{3}\left[3 \ln \left(1-e^{-\beta \Theta_{l}}\right)-4 D\left(\beta \Theta_{l}\right)\right]-\frac{2 N}{3}\left[3 \ln \left(1-e^{-\beta \Theta_{t}}\right)-4 D\left(\beta \Theta_{t}\right)\right], \\
E=\frac{3 N}{8}\left(\Theta_{l}+2 \Theta_{t}\right)+N T D\left(\beta \Theta_{l}\right)+2 N T D\left(\beta \Theta_{t}\right) .
\end{gathered}
$$

Теплоемкость $C_{V}=T(\partial S / \partial T)$, с учетом (35), определяется формулой

$$
C_{V}=N\left[4 D\left(\beta \Theta_{l}\right)-\frac{3 \beta \Theta_{l}}{e^{\beta \Theta_{l}}-1}\right]+2 N\left[4 D\left(\beta \Theta_{t}\right)-\frac{3 \beta \Theta_{t}}{e^{\beta \Theta_{t}}-1}\right] .
$$

Здесь энергии Дебая определены так же, как и выше $\Theta_{t}=\hbar c_{t} k_{D}, \Theta_{l}=\hbar c_{l} k_{D}$, но теперь скорости выражаются через инварианты тензора модулей упругости кристалла оптимальными соотношениями (28). Если в формулах (34)-(37) формально положить энергии Дебая равными, то получим соответствующие формулы стандартной теории Дебая. Однако, поскольку в изотропной среде справедливо неравенство $c_{l} / c_{t}>\sqrt{4 / 3}$, то такое же неравенство $\Theta_{l} / \Theta_{t}>\sqrt{4 / 3}$ справедливо и для энергий Дебая, а потому эти энергии не могут быть равными.

Поскольку более привычно вместо двух энергий Дебая пользоваться одной энергией, введем вместо двух параметров размерности энергии $\Theta_{t}, \Theta_{l}$ единую среднюю энергию Дебая $\Theta$ и один безразмерный параметр, в качестве которого удобно выбрать угол $\chi$ :

$$
\Theta^{2}=\frac{1}{3}\left(2 \Theta_{t}^{2}+\Theta_{l}^{2}\right), \quad \sin \chi=\sqrt{\frac{2}{3}} \frac{\Theta_{t}}{\Theta}, \quad \cos \chi=\frac{1}{\sqrt{3}} \frac{\Theta_{l}}{\Theta}, \quad \operatorname{tg} \chi=\sqrt{2} \frac{\Theta_{t}}{\Theta_{l}} .
$$

Определенная таким образом средняя энергия Дебая $\Theta$ соответствует усреднению скоростей (32). Вследствие приведенного выше неравенства $\Theta_{l} / \Theta_{t}>\sqrt{4 / 3}$ для отношения энергий Дебая, тангенс введенного в (38) угла $\chi$ принимает максимальное значение при угле $\chi_{m}$, который определяется условием $\operatorname{tg} \chi_{m}=\sqrt{3 / 2}$, откуда $\chi_{m}=0,886$ и, соответственно, $\cos \chi_{m}=\sqrt{2 / 5}, \sin \chi_{m}=\sqrt{3 / 5}$. Таким образом, угол может изменяться в пределах $0 \leq \chi<\chi_{m}$. Приведем также выражения для определенных в (38) параметров через инварианты тензора упругости кристалла:

$$
\Theta^{2}=\frac{\hbar^{2} k_{D}^{2}}{9 \rho} \lambda_{B}, \quad \sin ^{2} \chi=\frac{\left(3 \lambda_{B}-\lambda_{A}\right)}{5 \lambda_{B}}, \quad \cos ^{2} \chi=\frac{\left(2 \lambda_{B}+\lambda_{A}\right)}{5 \lambda_{B}} .
$$


Рассмотрим более детально поведение теплоемкости (37) с температурой. Как известно, в стандартной теории Дебая теплоемкость есть универсальная функция отношения $T / \Theta_{D}$ [5]. По этой модели теплоемкости всех тел, находящихся в соответственных состояниях, т.е. обладающих одинаковыми $T / \Theta_{D}$, должны быть одинаковыми. Для реальных тел этот закон, разумеется, не выполняется. В предлагаемой двухпараметрической модели, как видим, закон соответственных состояний не имеет места, поскольку теплоемкость зависит также от параметра $\chi$, который различен для разных тел (39). При высоких температурах $T>>$ формула для теплоемкости внешне имеет тот же вид, что в обычной теории

$$
C_{V}=3 N\left[1-\frac{1}{20}\left(\frac{\Theta}{T}\right)^{2}\right] .
$$

Здесь средняя энергия Дебая определена соотношениями (38), (39), а зависимость от параметра $\chi$ в этом приближении выпадает. При низких температурах $T<<\Theta$, как и в обычной теории, сохраняется кубическая зависимость от температуры

$$
C_{V}=\frac{12}{5} \pi^{4} N \cdot f(\chi)\left(\frac{T}{\Theta}\right)^{3},
$$

однако в данном случае коэффициент зависит также от параметра $\chi$ через функцию

$$
f(\chi) \equiv \frac{1}{3^{5 / 2}}\left(\frac{1}{\cos ^{3} \chi}+\frac{2^{5 / 2}}{\sin ^{3} \chi}\right)
$$

и оказывается различным для разных веществ. Влияние второго параметра $\chi$ наиболее существенно проявляется при низких температурах. При высоких температурах главное отличие двухпараметрической теории состоит в том, что здесь иначе определена средняя энергия Дебая (38), (39) и, при этом, она связана с инвариантами тензора упругости кристалла определенной симметрии (39).

Приведем также выражение для плотности фононных уровней, которая в данном случае определяется формулой

$$
g(\omega)=\int \frac{d \mathbf{k}}{(2 \pi)^{3}}\left[\delta\left(\omega-c_{l} k\right)+2 \delta\left(\omega-c_{t} k\right)\right] .
$$

В результате имеем

$$
g(\omega) \cdot 2 \pi^{2}=\left\{\begin{array}{lc}
\omega^{2}\left(1 / c_{l}^{3}+2 / c_{t}^{3}\right), & \omega<k_{D} c_{t}, \\
\omega^{2} / c_{l}^{3}, & k_{D} c_{t}<\omega<k_{D} c_{l}, \\
0, & \omega>k_{D} c_{l} .
\end{array}\right.
$$

Вид плотности фононных состояний показан на рис. 2. Из-за наличия двух фононных ветвей у функции $g(\omega)$ возникает скачёк при частоте $\omega=k_{D} c_{t}$, и, поэтому, структура плотности состояний оказывается сложнее, чем в стандартной модели. Как известно [8,9], и в плотности фононных состояний реальных кристаллов имеются особенности Ван Хова, так что двухпараметрическая модель в этом отношении ближе к реальным кристаллам.

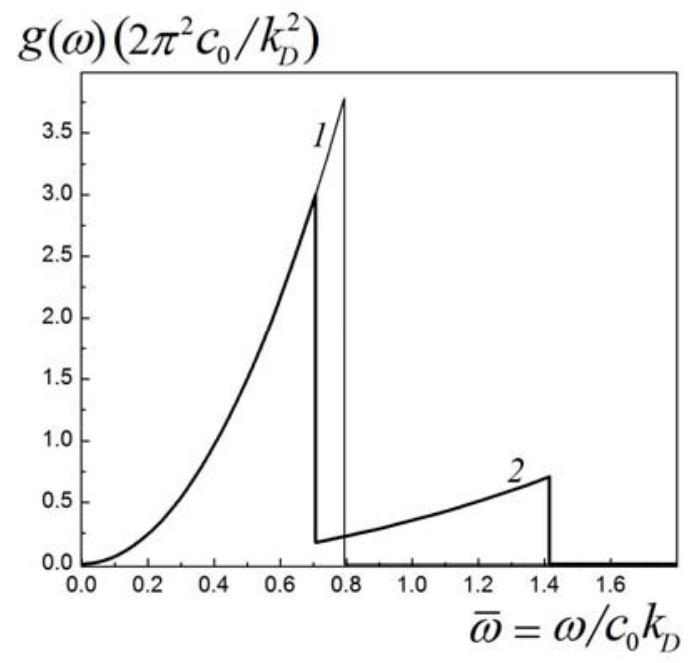

Рис. 2. Плотности фононных состояний $g(\omega)\left(2 \pi^{2} c_{0} / k_{D}^{2}\right)$, как функции частоты $\bar{\omega}=\omega / c_{0} k_{D}$;

1 - однопараметрическая модель, 2 - двухпараметрическая модель. Рассчитано при $\lambda / \mu=2$. 


\section{КРИСТАЛЛЫ КУБИЧЕСКОЙ СИНГОНИИ}

В качестве примера рассмотрим применение двухпараметрической теории Дебая к кристаллам кубической сингонии, которые наиболее близки к изотропной среде и характеризуются тремя модулями упругости. В этом случае два инварианта через три модуля упругости кубического кристалла в стандартных обозначениях [2] даются выражениями $\lambda_{A}=3 c_{11}+6 c_{12}, \lambda_{B}=3 c_{11}+6 c_{44}$, а аппроксимирующие коэффициенты Ламэ:

$$
\tilde{\lambda}=\frac{1}{5}\left(c_{11}+4 c_{12}-2 c_{44}\right), \quad \tilde{\mu}=\frac{1}{5}\left(c_{11}-c_{12}+3 c_{44}\right) .
$$

Значения этих величин для некоторых кубических кристаллов при низких температурах приведены в таблице 1.

Таблица 1.

Значения адиабатических модулей упругости вольфрама, меди и свинца при низких температурах [8], инвариантов $\lambda_{A}, \lambda_{B}$ и аппроксимирующих коэффициентов Ламэ (45) (в единицах $10^{12} \mathrm{dyn} / \mathrm{cm}$ ).

\begin{tabular}{|c|c|c|c|c|c|c|c|}
\hline Кристалл & $c_{11}$ & $c_{12}$ & $c_{44}$ & $\lambda_{A}$ & $\lambda_{B}$ & $\tilde{\lambda}$ & $\tilde{\mu}$ \\
\hline $\mathrm{W}$ & 5,326 & 2,049 & 1,631 & 28,272 & 25,764 & 2,052 & 1,634 \\
\hline $\mathrm{Cu}$ & 1,762 & 1,249 & 0,818 & 12,78 & 10,194 & 1,024 & 0,593 \\
\hline $\mathrm{Pb}$ & 0,555 & 0,454 & 0,194 & 4,389 & 2,829 & 0,397 & 0,137 \\
\hline
\end{tabular}

В таблице 2 приведены плотность, поперечная и продольная скорости фононов, вычисленные с использованием данных таблицы 1 и средние скорости, определённые формулами (31) и (32).

Таблица 2.

Значения плотности $\rho\left(\mathrm{g} / \mathrm{cm}^{3}\right)$, поперечной $c_{t}$ и продольной $c_{l}$ скоростей $(28)$, а также усредненных скоростей $c_{D}(31)$ и $c_{0}(32)$ (в единицах $10^{6} \mathrm{~cm} / \mathrm{s}$ ).

\begin{tabular}{|c|c|c|c|c|c|}
\hline Кристалл & $\rho$ & $c_{t}$ & $c_{l}$ & $c_{D}$ & $c_{0}$ \\
\hline $\mathrm{W}$ & 1,551 & 0,292 & 0,524 & 0,225 & 0,385 \\
\hline $\mathrm{Cu}$ & 9,018 & 0,257 & 0,495 & 0,199 & 0,355 \\
\hline $\mathrm{Pb}$ & 11,60 & 0,109 & 0,240 & 0,085 & 0,165 \\
\hline
\end{tabular}

В таблице 3 даны волновое число Дебая $k_{D}$, параметр $\chi(38)$, значение функции $f(\chi)(42)$ и температуры Дебая, вычисленные по формулам $\Theta_{t}=\hbar c_{t} k_{D}, \Theta_{l}=\hbar c_{l} k_{D}, \Theta_{D}=\hbar c_{D} k_{D}, \Theta \equiv \Theta_{0}=\hbar c_{0} k_{D}$, где величины скоростей взяты из таблицы 2, а также экспериментальное низкотемпературное значение температуры Дебая $\Theta_{D \exp }[8]$.

Таблица 3.

Волновое число $k_{D}\left(\right.$ в $\left.10^{8} \mathrm{~cm}^{-1}\right)$, температуры Дебая в градусах Кельвина (К).

\begin{tabular}{|c|c|c|c|c|c|c|c|c|}
\hline Кристалл & $k_{D}$ & $\Theta_{t}$ & $\Theta_{l}$ & $\Theta=\Theta_{0}$ & $\Theta_{D}$ & $\Theta_{\text {Dexp }}$ & $\chi$ & $f(\chi)$ \\
\hline $\mathrm{W}$ & 1,551 & 346 & 621 & 456 & 266 & 400 & 0,665 & 1,676 \\
\hline $\mathrm{Cu}$ & 1,717 & 337 & 650 & 466 & 261 & 343 & 0,632 & 1,882 \\
\hline $\mathrm{Pb}$ & 1,263 & 105 & 232 & 159 & 82 & 105 & 0,569 & 2,427 \\
\hline
\end{tabular}

Как видим, вычисленные в стандартной однопараметрической модели по величинам упругих постоянных температуры Дебая $\Theta_{D}$, оказываются существенно меныше измеренных температур Дебая $\Theta_{D \exp }$. Расчет по величинам упругих постоянных температуры Дебая $\Theta$ в предложенной двухпараметрической модели даёт значения, большие чем $\Theta_{D \exp }$. Введение в теорию второго параметра наиболее заметно влияет на расчёт термодинамических величин при низких температурах. На рис. 3 показаны расчетные температурные зависимости теплоёмкости меди при низких температурах. Расчёты выполнены с использованием аппроксимированных значений модулей упругости для однопараметрической (кривая 1) и двухпараметрической (кривая 2) моделей. Кривая 3 построена по экспериментальному значению температуры 
Дебая. Относительная ошибка $\delta=\left(C_{V}-C_{V \exp }\right) / C_{V \exp }$ в однопараметрической модели для меди $\delta_{1}=1,27$. В двухпараметрической модели расхождение с экспериментальным значением оказывается значительно меньшим: $\delta_{2}=0,25$. Величины этих же отклонений для вольфрама $\delta_{1}=2,7$ и $\delta_{2}=0,57$, а для свинца $\delta_{1}=1,1$ и $\delta_{2}=0,3$. Как видим расчет в однопараметрической модели даёт большое расхождение с экспериментальными данными. В тоже время двухпараметрическая модель приводит к значительно лучшему согласию с экспериментом и может быть использована для теоретических оценок температуры Дебая по величинам модулей упругости кристалла.

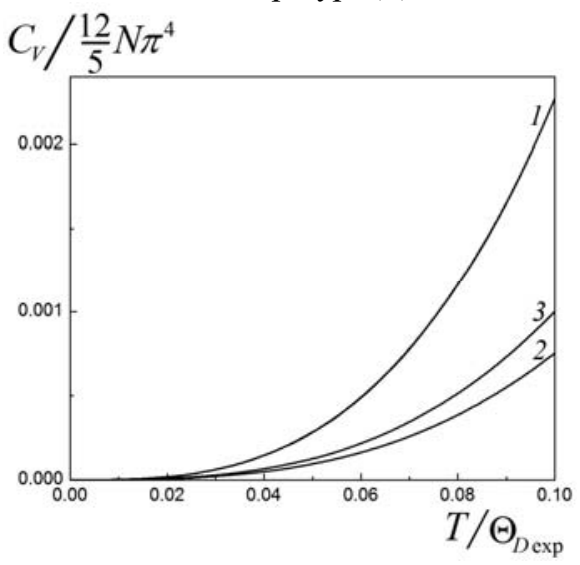

Рис. 3. Температурная зависимость теплоемкости меди при низких температурах:

1 - расчет через аппроксимированные модули упругости по однопараметрической модели, 2 - аналогичный расчет по двухпараметрической модели, 3 - зависимость в стандартной модели, где температура Дебая рассматривается как

феноменологический параметр. Температура Дебая для $\mathrm{Cu} \Theta_{D \exp }=343 \mathrm{~K}$.

\section{ВЫВОДЫ}

Показано, что предложенный ранее [2] метод описания упругих свойств кристаллов на основе сравнения с изотропной средой вытекает из требования максимальной близости свободных энергий кристалла и изотропной среды. В работе предложена модель изотропной упругой среды, аналогичная стандартной модели Дебая, где учитывается существование как поперечных, так и продольных фононов. В этой модели, помимо энергии Дебая вводится дополнительный параметр, так что закон соответственных состояний, характерный для обычной теории Дебая перестает выполняться. Расчет теплоемкости при низких температурах с использованием аппроксимированных модулей упругости приводит к значительно лучшему согласию с экспериментальными данными, чем для обычной теории, использующей среднюю скорость фононов.

Существует две крайние точки зрения на модель Дебая. Зачастую модели Дебая придают излишне общий смысл и при обработке экспериментальных данных подгоняют наблюдаемые величины к соотношениям этой теории, полагая, что энергия Дебая зависит от температуры. Противоположная точка зрения состоит в том, что соотношения теории Дебая рассматриваются как грубые интерполяционные формулы [1,9]. Модель Дебая, разумеется, является весьма приближенной и простой (в чем и состоит её ценность) моделью твердого тела, но, по нашему мнению, ее значение не сводится только к возможности построения единой интерполяционной формулы, которая правильно бы описывала поведение тела в пределе низких и высоких температур. Эта модель, как показано, допускает дальнейшее развитие и, в частности, обобщение с учетом взаимодействия фононов [6,7], а также может быть распространена для описания поверхностных явлений в твердом теле.

\section{СПИСОК ЛИТЕРАТУРЫ}

1. Landau L.D., Lifshic E.M. Teoriya uprugosti [Theory of elasticity]. - M.: Nauka, 1987. - 246 p. (in Russian)

2. Fedorov F.I. Teoriya uprugikh voln v kristallakh [The theory of elastic waves in crystals]. - M.: Nauka, 1965. - 388 p. (in Russian)

3. Akhiezer A.I., Aleksin V.F., Khodusov V.D. Gazodinamika kvazichastitc. I. Obshchaya teoriya [Gas dynamics of quasiparticles. General theory] // FNT. - 1994. - Vol. 20. - No.12. - P. 1199-1238. (in Russian)

4. Aleksin V.F., Belyaev N.R., Khodusov V.D. Model' privedennogo izotropnogo kristalla otnositel'no modulei uprugosti [The model of a reduced isotropic crystal with respect to the elastic moduli] // The Journal of Kharkiv National University, physical series "Nuclei, Particles, Fields". - 1999. - No.438. - Iss.1(5). - P.39-42. (in Russian)

5. Landau L.D., Lifshic E.M. Statisticheskaya fizika. Chast' I [Statistical physics. Part I]. - M.: Nauka, 1976. - 584 s. (in Russian)

6. Poluektov Yu.M. Samosoglasovannoe opisanie sistemy vzaimodeistvuyushchikh fononov [Self-consistent description of a system of interacting phonons] // FNT. - 2015. - T. 41, No.11. - S. 1081-1090. [Low Temperature Physics. - 2015. - Vol. 41. - No.11. - P. 922-929]. (in Russian)

7. Poluektov Yu.M. Samosoglasovannoe opisanie vzaimodeistvuyushchikh fononov v kristallicheskoi reshetke [Self-consistent description of interacting phonons in the crystal lattice] // East European Journal of Physics. - 2016. - Vol.3. - No.3. - P. 35-46. (in Russian)

8. Kittel' Ch. Vvedenie v fiziku tverdogo tela [Introduction to Solid State Physics]. - M.: Nauka, 1978. - 792 p. (in Russian)

9. Ashkroft N., Mermin N. Fizika tverdogo tela. Tom 2 [Solid State Physics. Volume 2]. - M.: Mir, 1979. - 422 p. (in Russian) 